\title{
ESPAÇAMENTO ENTRE PLANTAS NO DESEMPENHO DE JARDIM CLONAL DE CULTIVARES DE OLIVEIRA
}

\section{SPACEMENT BETWEEN PLANTS IN THE CLONAL GARDEN PERFORMANCE OF OLIVE TREE CULTIVARS}

\author{
Adelson Francisco de OLIVEIRA ${ }^{1}$ \\ João VIEIRA NETO \\ Fabíola VILLA ${ }^{3}$ \\ Luiz Fernando de Oliveira da SILVA ${ }^{4}$
}

\begin{abstract}
RESUMO
O trabalho teve por objetivo avaliar o desempenho de jardim clonal de cultivares e densidade de plantas de oliveira visando sua propagação por estaquia. O jardim clonal foi instalado em março de 2008, o plantio feito em sulcos com $40 \mathrm{~cm}$ de profundidade e quatro espaçamentos entre plantas e o primeiro corte foi realizado em fevereiro de 2009. Foram avaliadas três cultivares de oliveira ('Arbequina', 'Grappolo 541' e 'Ascolano 315') e quatro espaçamentos entre plantas (fevereiro de 2009). O delineamento utilizado foi de blocos ao acaso com parcelas subdivididas no espaço e cinco repetições. As parcelas foram constituídas de três linhas de um metro de comprimento, espaçadas entre si em um metro, com duas, três, quatro e cinco plantas em cada linha. Para avaliação, as plantas foram podadas a $20 \mathrm{~cm}$ de altura do solo, após 12 meses de cultivo, sendo avaliados a altura da planta $(\mathrm{m})$, diâmetro do tronco $(\mathrm{mm})$, comprimento médio de ramos $(\mathrm{m})$, rendimento em número de estacas e massa verde total acumulada $(\mathrm{kg})$. Em todas as características, melhores resultados foram observados em 'Ascolano 315', sendo maior comprimento médio dos ramos com densidade de 3 e 5 plantas por metro.
\end{abstract}

Palavras-chave: estaquia; produção de mudas; Olea europaea.

\begin{abstract}
The work had for objective to evaluate the performance of cultivar olive trees of clonal garden aimed this propagation for cutting. The clonal garden was installed in March 2008, with ridges of $40 \mathrm{~cm}$ depth and four spacement between plants and the first cut had been evaluated in February 2009. Were evaluated three olive varieties ('Arbequina', 'Grappolo 541' and 'Ascolano 315') and four spacement between plants (February 2009). The experimental design was blocks with parcels subdivided in the space and five repetitions. The parcels had been constituted of three lines of one meter length, spaced between itself in one meter, with two, three, four and five plants in each line. For evaluation, the plants had been trimmed the $20 \mathrm{~cm}$ height of ground, after 12 months of culture, being evaluated the plant height $(\mathrm{m})$, diameter of plant $(\mathrm{mm})$, average length of branches $(\mathrm{m})$, income in number of cutting and accumulated total green mass $(\mathrm{kg})$. In almost all characteristics, better resulted had been observed in 'Ascolano 315', being bigger average branches length with density of 3 and 5 plants for meter.
\end{abstract}

Key-words: cutting; production of seedlings; Olea europaea.

\footnotetext{
${ }^{1}$ D.Sc., Pesquisador da EPAMIG-CTSM. Campus Universitário da UFLA, Caixa Postal 176, Rodovia Lavras/IJACI, km 02, CEP 37200-000, Lavras, MG. E-mail: adelson@epamig.br

${ }^{2}$ D.Sc., Pesquisador da EPAMIG. Bairro Vargedo, CEP 35517-000, Maria da Fé, MG, Bolsista Fapemig. E-mail: joaovieira@epamig.br

${ }^{3}$ Pós-doutoranda em Fitotecnia, bolsista FAPEMIG/EPAMIG, Bairro Vargedo, CEP: 37517-000, Maria da Fé, MG. E-mail: fvilla2003@libero.it

${ }^{4}$ Eng. Agr. Bolsista FAPEMIG/EPAMIG, Bairro Vargedo, CEP 37517-000, Maria da Fé, MG. E-mail: luizfernando.agronomia@gmail.com
} 
OLIVEIRA, A. F. et al. Espaçamento entre plantas no desempenho...

\section{INTRODUÇÃO}

A oliveira (Olea europaea L., oleaceae), teve seu cultivo iniciado a quatro mil anos a.C. próximo ao Mar Morto, região que abrange a Síria, Líbano e Israel, expandindo-se para o Ocidente pela bacia do Mediterrâneo. Atualmente é cultivada em praticamente todos os continentes, no entanto, sua produção comercial está concentrada em países da União Européia, sendo essa região, a maior produtora mundial (Rallo, 2008).

O cultivo de oliveiras no Brasil, com finalidade comercial, é uma atividade agrícola recente e em expansão. Tendo como base o volume de importação de azeitona praticado em 2008, 214 mil t (Brasil, 2007), estima-se um mercado potencial de mudas de aproximadamente 11 milhões de unidades, exigindo da pesquisa informações técnicas para sua produção.

A implantação de jardim clonal com o objetivo de fornecer material propagativo é considerado vantajoso, pois além de permitir a coleta de ramos durante todo o ano, as plantas podem apresentar caráter juvenil e vigor vegetativo, características favoráveis ao enraizamento (Caballero, 1981). Segundo Hackett (1987) a juvenilidade é um fator importante em plantas lenhosas por afetar a capacidade de propagação vegetativa, além de proporcionar variações nas taxas e formas de crescimento, na qualidade e rapidez na formação de raízes, e também mudanças fisiológicas e bioquímicas, com a transição para o estágio maduro.

A produção de mudas é um importante elo da cadeia produtiva, pois se constitui no primeiro passo para a implantação da cultura da oliveira. A obtenção de mudas de qualidade, além de garantir uniformidade e idoneidade varietal, é um fator que influencia toda a vida do pomar, permitindo maximizar os efeitos de clima e solo e principalmente de tratos culturais adotados à cultura.

As densidades utilizadas em olivais nos países, grandes produtores, são muito variáveis, segundo as zonas de cultivo (Alvarenga et al., 2006). Em plantios convencionais de oliveira no Brasil, costuma-se utilizar espaçamento de $4 \mathrm{~m}$ entre plantas e $6 \mathrm{~m}$ entre fileiras (Vieira Neto et al., 2008).

Diante do exposto, o objetivo do presente trabalho foi avaliar o desempenho de jardim clonal de cultivares de oliveira, em diferentes densidades, visando sua propagação por estaquia.

\section{MATERIAL E MÉTODOS}

O trabalho iniciou-se em fevereiro/2008, na Fazenda Experimental da Epamig (FEMF), localizada no município de Maria da Fé, microregião da Serra da Mantiqueira, sul do Estado de Minas Gerais.

O município de Maria da Fé apresenta uma classificação climática, segundo Köppen, do tipo Cwb, ou seja, clima temperado chuvoso (mesotérmico), também chamado subtropical de altitude. Caracteriza-se por apresentar temperatura média anual em torno de $17^{\circ} \mathrm{C}$, uma temperatura média máxima de $23,3{ }^{\circ} \mathrm{C}$ e uma mínima média de $10,1{ }^{\circ} \mathrm{C}$, precipitação anual de $1.738,6 \mathrm{~mm}$ e altitude média de $1.276 \mathrm{~m}$.

Os jardins clonais foram instalados em fevereiro de 2008, em área previamente selecionada e a acidez do solo corrigida de acordo com recomendação de análise de fertilidade (Tabela 1) para pH em torno de 6,0, utilizando-se calcário dolomítico (PRNT - Poder Relativo de Neutralização Total, 100\%). Foram avaliadas, nesta área, três cultivares de oliveira (Grappolo 541, Ascolano 315 e Arbequina) em quatro densidades de plantio ( 2 plantas $\mathrm{m}^{-1}, 3$ plantas $\mathrm{m}^{-1}, 4$ plantas $\mathrm{m}^{-}$ ${ }^{1}$ e 5 plantas $\mathrm{m}^{-1}$.

TABELA 1 - Análise de fertilidade do solo. EPAMIG, Maria da Fé, MG, 2009.

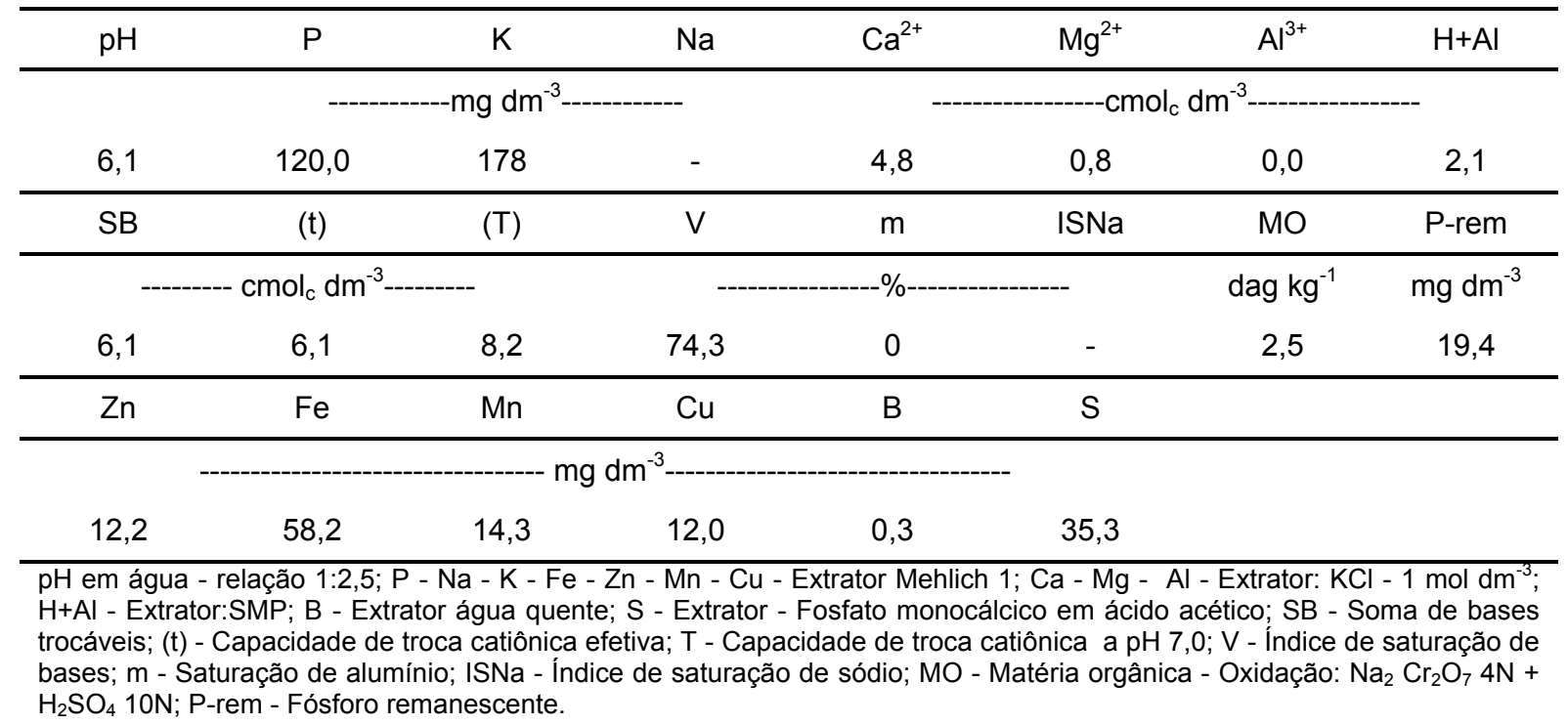


OLIVEIRA, A. F. et al. Espaçamento entre plantas no desempenho...

O delineamento estatístico utilizado foi o blocos ao acaso com parcelas subdivididas com cinco repetições, subparcelas no espaço e três cultivares de oliveira. As parcelas experimentais foram constituídas de três linhas de $1 \mathrm{~m}$ de comprimento, espaçadas entre si em $1 \mathrm{~m}$, com duas, três, quatro e cinco plantas em cada linha.

As mudas de oliveira utilizadas no ensaio foram obtidas por meio de enraizamento de estacas semilenhosas tratadas com Ácido Indolbutírico (AIB) e mantidas em câmara de nebulização intermitente. Os plantios foram realizados em sulcos com $40 \mathrm{~cm}$ de profundidade, as quais receberam, com antecedência de 60 dias, $15 \mathrm{~cm}^{3} \mathrm{dm}^{-3}$ da mistura esterco de curral + esterco de galinha, na proporção de 1:1 (devidamente incorporado), enriquecidos com $1,5 \mathrm{~kg}$ de superfosfato simples em um metro linear de sulco. Em todas as parcelas experimentais foram aplicados, mensalmente, via pulverização foliar, adubação de macro e micronutrientes na concentração de $14 \%$ de $\mathrm{N}$ (amoniacal 0,7\%, nítrica $8 \%$ e amídica $5,3 \%), 4 \%$ de $\mathrm{P}\left(\mathrm{P}_{2} \mathrm{O}_{5}\right), 20 \%$ de $\mathrm{K}$ $\left(\mathrm{K}_{2} \mathrm{O}\right), 2 \%$ de $\mathrm{Mg}, 2 \%$ de $\mathrm{B}$ e $0,05 \%$ de $\mathrm{Mn}$.

As plantas do jardim clonal receberam irrigação localizada sempre que necessário, em quantidades suficientes de acordo com a necessidade da cultura para manter o solo umedecido (capacidade de campo). Foram realizados os tratamentos fitossanitários usuais, utilizando produtos específicos, de acordo com a necessidade, mantendo também as áreas livres de ervas daninhas.

Para avaliação as plantas foram podadas a $20 \mathrm{~cm}$ de altura do solo, sendo avaliados a altura da planta $(\mathrm{m})$, diâmetro do tronco $(\mathrm{mm})$ a $20 \mathrm{~cm}$ de altura do solo, comprimento médio de ramos $(\mathrm{m})$, rendimento em número de estacas com quatro nós e dois pares de folhas (em torno de $12 \mathrm{~cm}$ de comprimento) e massa verde total acumulada ( $\mathrm{kg})$.

Os dados coletados foram avaliados e os resultados obtidos foram submetidos a análise de variância utilizando o programa Sisvar (Ferreira, 2000). Foi aplicada a análise de variância, com teste $F$ para verificar os efeitos principais e da interação entre os fatores e confirmado efeito significativo foi aplicado o teste de Tukey para comparar as médias. Para o número de estacas por planta foi aplicado a transformação $(x+0,5)^{1 / 2}$, com finalidade de proporcionar a normalidade dos erros experimentais. Em todas as análises foram adotados $5 \%$ de probabilidade de erro experimental.

\section{RESULTADOS E DISCUSSÃO}

$\mathrm{Na}$ Tabela 2 encontram-se os resultados da análise de variância que indicaram significância para o efeito nas cultivares estudadas e densidade de plantio.

TABELA 2 - Análise de variância para altura média das plantas ( $A M, m)$, diâmetro médio do tronco (DMT, mm), massa verde total (MVT, $\mathrm{kg}$ ), comprimento médio dos ramos (CMR, $\mathrm{m}$ ) e número médio de estacas (NME) de oliveira em jardim clonal. EPAMIG, Maria da Fé, MG, 2009.

\begin{tabular}{|c|c|c|c|c|c|c|}
\hline \multirow{2}{*}{ FV } & \multirow[t]{2}{*}{$\overline{G L}$} & \multicolumn{5}{|c|}{ QM } \\
\hline & & $\mathrm{AM}(\mathrm{m})$ & DMT $(\mathrm{mm})$ & MVT (kg) & CMR (m) & NME \\
\hline DP & 3 & $0,035^{\text {n.s. }}$ & $24,541^{\text {n.s. }}$ & $0,026^{\text {n.s. }}$ & $0,114^{*}$ & $1,307^{\text {n.s. }}$ \\
\hline Cultivar & 2 & $0,494^{*}$ & $171,103^{*}$ & $0,184^{*}$ & $0,255^{*}$ & $37,051^{*}$ \\
\hline Bloco & 3 & 0,035 & 24,541 & 0,025 & 0,114 & 1,307 \\
\hline DP $x$ cultivar & 6 & $0,145^{\text {n.s. }}$ & $23,341^{\text {n.s. }}$ & $0,041^{\text {n.s. }}$ & $0,087^{\text {n.s. }}$ & $2,559^{\text {n.s. }}$ \\
\hline Erro & 21 & 0,138 & 13,369 & 0,024 & 0,037 & 2,873 \\
\hline Total & 35 & & & & & \\
\hline CV (\%) & 18,46 & 18,46 & 13,99 & 18,39 & 16,00 & 20,91 \\
\hline
\end{tabular}

$\mathrm{FV}=$ fator de variação, $\mathrm{GL}$ = graus de liberdade, $\mathrm{QM}=$ quadrados médios, $\mathrm{DP}=$ densidade de plantio. *significativo a $5 \%$ de probabilidade, n.s. = não significativo

Observa-se na Tabela 3 que a altura das plantas foi influenciada somente pelas cultivares estudadas, embora não diferindo estatisticamente entre as mesmas, onde a maior altura média foi verificada em 'Ascolano 315', alcançando 2,22 m de altura.

Em relação ao diâmetro médio do tronco, a cultivar Ascolano 315 superou em 1,32 vezes o diâmetro do tronco da 'Arbequina' e a 'Grappolo
541 ' em 1,2 vezes, sendo $28,91 \mathrm{~mm}$ na 'Ascolano 315'. Segundo Del Rio \& Caballero (2006), a altura e o diâmetro da planta são parâmetros que fazem referência ao vigor da árvore de oliveira, definindo o seu tamanho definitivo. Esses autores atribuem à cultivar como sendo a principal causa da variabilidade observada para esse critério agronômico. 
OLIVEIRA, A. F. et al. Espaçamento entre plantas no desempenho...

TABELA 3 - Altura média (AM, m), diâmetro médio do tronco (DMT, mm), massa verde total (MVT, $\mathrm{kg}$ ), comprimento médio dos ramos (CMR, $m$ ) e número médio de estacas (NME) de três cultivares de oliveira em jardim clonal, no primeiro ano de corte. EPAMIG, Maria da Fé, 2009.

\begin{tabular}{lccccc}
\hline Cultivares & AM $(\mathrm{m})$ & DMT $(\mathrm{mm})$ & MVT $(\mathrm{kg})$ & CMR $(\mathrm{m})$ & NME \\
\hline Ascolano 315 & $2,22 \mathrm{a}^{*}$ & $28,91 \mathrm{a}$ & $0,97 \mathrm{a}$ & $1,33 \mathrm{a}$ & $9,28 \mathrm{a}$ \\
Grappolo 541 & $1,99 \mathrm{a}$ & $27,68 \mathrm{a}$ & $0,83 \mathrm{~b}$ & $1,24 \mathrm{a}$ & $8,95 \mathrm{a}$ \\
Arbequina & $1,82 \mathrm{a}$ & $21,84 \mathrm{~b}$ & $0,73 \mathrm{~b}$ & $1,04 \mathrm{~b}$ & $6,09 \mathrm{~b}$ \\
\hline
\end{tabular}

${ }^{*}$ Letras minúsculas diferem entre si estatisticamente na coluna pelo teste de Tukey a $5 \%$ de probabilidade.

As variáveis agronômicas estudadas como altura de plantas, formato de frutos e vigor diferem significativamente para cultivares de mesa e para azeite (IOOC, 2007). O vigor de uma planta pode ser avaliado pela espessura $x$ largura $x$ altura da planta. Na cultivar Ascolano 315 esta característica foi bem mais alta que a nas outras cultivares estudadas. Esta cultivar é muito apreciada pela precocidade de produção, elevada produtividade, bom rendimento em ácido graxo e excelente qualidade do azeite produzido, porém com baixa estabilidade. O vigor da planta é reduzido, permitindo maior adensamento de cultivo (Coutinho et al., 2007).

Verificou-se na Tabela 3, maior acúmulo de massa verde total para a 'Ascolano 315' $(0,97$ $\mathrm{kg}$ ). A importância da seleção de matrizes pelo volume da parte aérea, ausência de patógenos e susceptibilidade a doenças, permitiu que a propagação vegetativa se tornasse o principal método de reprodução clonal de árvores selecionadas (Haag, 1983). Dentre estes métodos, a estaquia é, ainda, a técnica de maior viabilidade econômica para o estabelecimento de plantios clonais de espécies florestais (Paiva \& Gomes, 1993).

Para comprimento médio dos ramos, observou-se significância nas cultivares de oliveira estudadas e no espaçamento entre plantas, separadamente (Tabela 3). Com 1,33 m de comprimento médio de ramos, a 'Ascolano 315' se sobressaiu, apesar de não diferir estatisticamente da 'Grappolo 541' (Tabela 3). Na Tabela 4, observase que o comprimento médio dos ramos foi maior nas densidades de plantio com 3 e 5 plantas $\mathrm{m}^{-1}$, alcançando respectivamente 1,31 e $1,29 \mathrm{~cm}$. A cultivar Arbequina, devido ao seu baixo vigor vegetativo tem sido amplamente utilizada em sistema de plantio superintensivo em todo mundo, especialmente na Argentina, Chile e Espanha (Ruis, 2007).

TABELA 4 - Comprimento médio dos ramos $(\mathrm{m})$ de três cultivares de oliveira plantadas, com diferentes espaçamentos entre plantas em jardim clonal, no primeiro ano de corte. EPAMIG, Maria da Fé, 2009.

\begin{tabular}{cc}
\hline Espaçamento entre plantas $(\mathrm{m})$ & Comprimento médio dos ramos $(\mathrm{m})$ \\
\hline 3 plantas $\mathrm{m}^{-1}$ & $1,31 \mathrm{a}$ \\
5 plantas $\mathrm{m}^{-1}$ & $1,29 \mathrm{a}$ \\
4 plantas $\mathrm{m}^{-1}$ & $1,16 \mathrm{~b}$ \\
2 plantas $\mathrm{m}^{-1}$ & $1,07 \mathrm{~b}$ \\
\hline
\end{tabular}

* Letras minúsculas diferem entre si estatisticamente na coluna pelo teste de Tukey a $5 \%$ de probabilidade.

Segundo Botelho (1998) a recomendação de um espaçamento em jardins clonais não pode ser generalizada, devendo-se levar em consideração a qualidade do jardim clonal, as características da espécie, os objetivos do manejo e as condições do mercado, bem como os métodos de colheita e/ou outros produtos. Para Sanquetta et al. (2003), a adoção de um determinado espaçamento de plantio tem muitas implicações no gerenciamento de jardins clonais. Segundo o mesmo autor, para uma mesma espécie e um mesmo jardim clonal, a escolha de um espaçamento implicará no número de tratos culturais a serem efetuados, na taxa de crescimento, no volume de matéria verde produzido, na idade de estagnação do crescimento, nas práticas de implantação, no manejo e exploração comercial, no volume da copa, nos custos de produção, dentre outros aspectos.

O número médio de estacas com quatro nós e dois pares de folhas retiradas da parte aérea das cultivares Ascolano 315 e Grappolo 541, no primeiro ano de corte, foi de 9,28 e 8,95, respectivamente, 1,53 e 1,47 vezes maior do que o número de estacas obtidas da cultivar Arbequina (Tabela 3). 
OLIVEIRA, A. F. et al. Espaçamento entre plantas no desempenho...

As diferenças nas médias apresentadas se deve ao fato da 'Ascolano 315' apresentar, hábito de crescimento mais intenso, quando comparada com outras cultivares recentemente estudadas no Brasil, como a 'Arbequina' e 'Grappolo 541'. Ao trabalhar com a caracterização morfológica de cultivares de oliveira do banco de germoplasma da Epamig em Maria da Fé, Oliveira et al. (2006) observaram que de todas as cultivares analisadas, a 'Ascolano 315' se destacou apresentando plantas com porte mais altas, sendo por isso considerada muito vigorosa. Estudos recentes de adaptação de cultivares no norte de Minas Gerais mostraram melhor rendimento agronômico em plantas de um ano dessa mesma cultivar (Oliveira et al., 2008).

Os resultados do primeiro ano aqui apresentados sugerem que se utilize a estaquia como método de propagação da oliveira e que os cortes devem se estender por mais alguns anos. Sendo assim deve-se dar continuidade nas avaliações experimentais até que se verifique queda significativa nos parâmetros avaliados, principalmente no rendimento em número de estacas, inviabilizando a manutenção do jardim clonal em nível comercial. Esta variável é um importante índice técnico, interferindo diretamente no planejamento de produção de mudas, sendo necessária no dimensionamento de viveiros de mudas.

É importante ressaltar que os efeitos marcantes do espaçamento deverão ser constatados em períodos de cortes sucessivos (segundo e terceiro ano), e que os mesmos deverão estar associados a desbastes e número médio de estacas para fins comerciais.
O comportamento das cultivares de oliveira referem-se às características intrínsecas aos genótipos estudados, não sendo motivo de exclusão de um em detrimento ao outro. Todos os materiais são importantes na implantação comercial da olivicultura no sul de Minas Gerais, pois as três cultivares estudadas destinam-se à mesa ('Ascolano 315'), produção de azeite ('Arbequina') e dupla finalidade ('Grappolo 541'). A utilização de jardins clonais para produção de estacas vem sendo amplamente utilizada em cafeeiros, frutíferas em geral e espécies florestais. Com o atual estudo, pretende-se empregar esta técnica em plantios comerciais brasileiros de oliveira.

\section{CONCLUSÕES}

1. A técnica de adensamento de jardins clonais mostrou-se eficiente para as cultivares de oliveira.

2. A cultivar Ascolano 315 apresenta melhores resultados do que as cultivares Arbequina e Grappolo 541, podendo ser utilizada em jardins clonais.

3. Independentemente da cultivar de oliveira, melhores resultados em jardins clonais foram verificados com densidade de plantio de 3 e 5 plantas $\mathrm{m}^{-1}$.

\section{AGRADECIMENTOS}

À Fundação de Amparo a Pesquisa do Estado de Minas Gerais (FAPEMIG) pelo apoio financeiro na execução desse trabalho. CAG APQ0497-3.08/07.

\section{REFERÊNCIAS}

1. ALVARENGA, A.A.; OLIVEIRA, A.F.; ABRAHÃO, E. Sistema de plantio para a cultura da oliveira. In: Azeitona e azeite de oliva: tecnologias de produção. Informe Agropecuário, v. 27, n. 231, p. 63-67, 2006.

2. BOTELHO, S.A. Espaçamento. In: SCOLFORO, J.R.S. Manejo florestal Lavras: UFLA/FAEPE, 1998 p. 381-405.

3. BRASIL. Indicadores da agropecuária, 2007. Disponível em: <http://www.conab.gov.br/conabweb/index.php? PAG $=212>$. Acesso em: 06 fev. 2009

4. CABALLERO, J. M. Multiplicación del olivo por etaquillado semileñoso bajo nebulización. Madrid: Instituto Nacional de Investigaciones Agrarias, 1981. 39 p. (Comunicaciones INIA, Serie Producción Vegetal, 31).

5. COUTINHO, E.F.; JORGE, R.O.; COSTA, V.B. Cultivares de oliveira. In: COUTINHO, E.F (Ed.). A cultura da oliveira Pelotas: EMBRAPA, 2007. p. 39-49.

6. DEL RIO, C.; CABALLERO, J.M. Caracterização de variedades de oliveira no Banco Mundial de Germoplasma de Córdoba - Espanha. Informe Agropecuário, v. 27, n. 231, p. 18-26, 2006.

7. FERREIRA, D. F. Análises estatísticas por meio do Sisvar para Windows versão 4.0. In: REUNIÃO ANUAL DA REGIÃO BRASILEIRA DA SOCIEDADE INTERNACIONAL DE BIOMETRIA, 45., 2000, São Carlos. Anais... São Carlos: UFSCar, 2000. p. 255-258.

8. HAAG, H.P. Nutrição mineral do Eucalyptus, Pinus, Araucaria e Gmelina no Brasil. Campinas: Fundação Cargil, 1983. $101 \mathrm{p}$

9. HACKETT, W.P. Donor plant maturation and adventitious root formation. In: DAVIES, T.D.; HAISSIG, B.E.; SANKHLA, N Adventitious root formation in cuttings. Portland: Discorides, 1987. p.11-28. (Advances in Plants Sciences Serie, 2).

10. IOOC. INTERNACIONAL OLIVE OIL CONCIL. Word olive oil figures: production. 2007. Disponível em: <http:// www.internationaloliveoil.org/web/aaingles/corp/AreasActivitie/economics/economics-oliveOilFigures.html>. Acesso em: 30 abril 2009

11. OLIVEIRA, A.F.; ANTUNES, L.E.C.; SCHUCH, M.W. Caracterização morfológica de cultivares de oliveira em coleção e considerações sobre o seu cultivo no Brasil. Informe Agropecuário, v. 231, n. 27, p. 55-62, 2006.

12. OLIVEIRA, A.F.; VIEIRA NETO, J.; GONÇALVES, E.D. et al. Rendimento agronômico de variedades de oliveira (Olea europaea L.) no norte de Minas Gerais. In: CONGRESSO BRASILEIRO DE FRUTICULTURA, 20., 2008, Vitória. Anais...Vitória: Sociedade Brasileira de Fruticultura, 2008.

13. PAIVA, H.N.; GOMES, J.M. Propagação vegetativa de espécies florestais. Viçosa: Universidade Federal de Viçosa, 1993. 40p

Scientia Agraria, Curitiba, v.11, n.4, p.317-322, July/Aug. 2010. 
OLIVEIRA, A. F. et al. Espaçamento entre plantas no desempenho...

14. RALLO, L. Variedades de olivo en España: una aproximación cronológica. In: RALLO, L.; BARRANCO, D.; CABALLERO, J. M.; DEL RÍO, C.; MARTÍN, A.; TOUS, J.; TRUJILLO, I. (Ed.). Variedades de olivo en España. Madri: Mundi-Prensa, 2008. cap. 1, p.17-44.

15. RUIS, X. Notas sobre plantaciones superintensivas de olivo em Chile. Olint, v. 1, n. 3, p. 12-15, 2007.

16. SANQUETTA, C.R.; MORA, A.L.; BORSATO, R.; VIDAL, M.A.S.; PEIXOTO, A.M.; CHIARANDA, R. Efeito do espaçamento de plantio em reflorestamento. II. Pinus taeda L. em Jaguariaíva-PR. Revista Acadêmica: ciências agrárias e ambientais, v. 1, n. 1, p. 55-61, 2003

17. VIEIRA NETO, J.; OLIVEIRA, A.F.; OLIVEIRA, N.C.; DUARTE, H.S.S.; GONÇALVES, E.D. Aspectos técnicos da cultura da oliveira. Belo Horizonte: EPAMIG, 2008. 56 p. (Boletim Técnico, 88).

Recebido em 18/11/2009 Aceito em 12/08/2010 